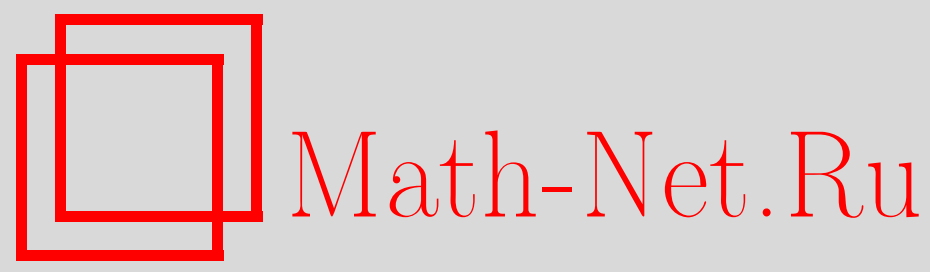

Г. И. Ивченко, К сравнению схем повторного и бесповторного выбора, Матем. заметки, 2003, том 73, выпуск 2, 195205

DOI: https://doi.org/10.4213/mzm178

Использование Общероссийского математического портала Math-Net.Ru подразумевает, что вы прочитали и согласны с пользовательским соглашением http://www . mathnet.ru/rus/agreement

Параметры загрузки:

IP: 44.207 .124 .84

26 апреля 2023 г., 05:48:47

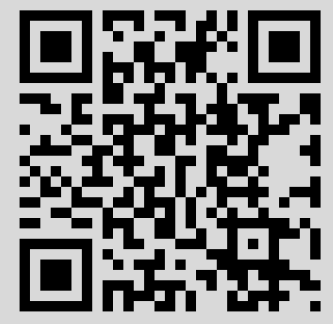




\section{К СРАВНЕНИЮ СХЕМ ПОВТОРНОГО И БЕСПОВТОРНОГО ВЫБОРА}

\section{Г.И. Ивченко}

Имеется урна с шарами $N$ различных цветов, каждый из которых представлен $a$ шарами. Из урны последовательно и равновероятно извлекается по одному шару, при этом каждый раз вынутый шар а) возвращается в урну (повторный выбор) или б) не возвращается (бесповторный выбор). Процесс извлечения шаров продолжается до тех пор, пока $k$ некоторых цветов впервые будут зафиксированы не менее $m$ раз каждый. Наблюдаемыми статистиками являются величины $\mu_{r}$ - количество цветов, представленных в момент остановки ровно $r$ раз каждый, $r=1,2, \ldots$ Исследуется асимптотическое при $N \rightarrow \infty$ поведение этих величин для каждой модели выбора и обсуждается проблема их использования для идентификации модели.

Библиографофия: 9 названий.

1. Введение. Пусть имеется урна с шарами $N$ различных цветов, при этом число шаров каждого цвета в начальньй момент равно $a$, так что всего в урне первоначально имеется $N a$ шаров. Далее начинается процесс последовательного и равновероятного извлечения шаров из урны либо по схеме выбора с возвращением (повторньй выбор), либо по схеме выбора без возвращения (бесповторньй выбор). В первом случае извлеченньй шар после фиксации его цвета возвращается обратно в урну, т.е. ее состав остается неизменным, и процесс извлечения может продолжаться неограниченно долго. Во втором случаешар в урну не возврашается, т.е. ее состав каждьй раз изменяется (уменьшается на единицу), и процесс извлечения может длиться максимум $N a$ шагов из-за исчерпания урны. Зададим случайный момент остановки испытаний (извлечения шаров) следующим образом: процесс извлечения шаров продолжается до тех пор, пока $k$ некоторых цветов впервые появятся по крайней мере $m$ раз каждьй. Здесь $k, 1 \leqslant k \leqslant N$, и $m \geqslant 1-$ управляемые параметры момента остановки; при этом в случае бесповторного выбора должно вьполняться естественное ограничение $m \leqslant a$ (больше, чем $a$ раз, никакой цвет не может наблюдаться), а при $k=N, m=a$ потребуется извлечь все $N a$ шаров. Во всех же остальных вариантах задания параметров $k$ и $m$ до момента остановки потребуется произвести некоторое случайное число испытаний, которое мы будем обозначать далее $\nu_{m}^{(0)}(N, k)$ для модели повторного и $\nu_{m}^{(-)}(N, k)$ для модели бесповторного выбора. Вообще, условимся в последующем снабжать все характеристики для модели повторного выбора индексом (0), а для модели бесповторного выбора индексом (-).

Обозначим, далее, $\mu_{r}^{(\cdot)}=\mu_{r}^{(\cdot)}(N, k, a, m)$ (здесь и в дальнейшем $(\cdot)$ либо 0, либо $(-)$ в соответствии со сказанным выше) число цветов в выборке, зафиксированных ровно $r$ раз каждьй, $r=1,2, \ldots$ В наборе статистик $\bar{\mu}^{(\cdot)}=\left\{\mu_{r}^{(\cdot)}, r \geqslant 1\right\}$ заключена 
вся информация о модели, и нашей задачей будет исследование их асимптотического $($ при $N \rightarrow \infty$ ) поведения и использование этой информации для идентификации модели. Отметим, что в модели бесповторного выбора набор $\bar{\mu}^{(-)}$имеет фиксированную размерность $a: \bar{\mu}^{(-)}=\left(\mu_{1}^{(-)}, \ldots, \mu_{a}^{(-)}\right)$, так как ни один из цветов не может повториться в выборке более $a$ раз, в то время как в модели повторного выбора возможно в принципе сколь угодно большое число повторений в выборке каждого из цветов, и, следовательно, соответствующий набор $\bar{\mu}^{(0)}$ не имеет фиксированной размерности: еевеличина $\kappa_{N}^{(0)}=\max \left\{r: \mu_{r}^{(0)}>0\right\}$ случайна. Подчеркнем также, что время ожидания до момента остановки является линейной функцией от статистик $\mu_{r}^{(\cdot)}: \nu_{m}^{(\cdot)}(N, k)=\sum_{r} r \mu_{r}^{(\cdot)}$, и представляет собой одну из важнейших характеристик модели, которой в литературе по дискретньп вероятностньм задачам традиционно уделяется большое внимание (см. [1] и библиограффию в ней).

Литература, посвященная обсуждаемой проблематике, весьма обширна. Вместе с тем, большинство соответствующих публикаций относятся к модели повторного выбора (см. обзор [2] и [3]), и лишь в последнее время были предложены общие методы исследования подобных задач, охватывающие и другие модели выбора, включая, в частности, и бесповторньй выбор (см. [1], [4]-[6]). В цитируемых работах развит аппарат предельных теорем для так называемых разделимых статистик, т.е. функций аддитивного вида от наблюдаемых частот исходов, частными случаями которых являются статистики $\mu_{r}$ и $\nu_{m}(N, k)$. Основьваясь на этих результатах и конкретизируя их в обсуждаемых аспектах, мы получаем здесь новые предельные теоремы, позволяющие под новьм углом зрения провести сравнительньй асимптотический анализ двух классических моделей случайного выбора из конечной совокупности.

Дальнейшая организация работы следующая. В п. 2 приводятся формулировки предельных теорем для статистик $\bar{\mu}^{(\cdot)}$ и $\nu_{m}^{(\cdot)}(N, k)$ в обеих моделях выбора, п. 3 посвящен обсуждению проблемы идентификации модели с использованием этих результатов; наконец, в п. 4 кратко излагается метод асимптотического анализа распределений рассматриваемых статистик.

2. Предельные теоремы. В дальнейшем предполагается, что число цветов $N \rightarrow \infty$, параметр $k$ момента остановки является функцией от $N: k=k(N)$ и изменяется тем или иным образом, а остальные параметры $a$ и $m$ фиксированы. Решаюшим фактором при этом является характер асимптотического поведения параметра $k$, что конкретизируется выделением трех областей его изменения:

1) $k$ фиксировано, $k \geqslant 1$ (левая область);

2) $s=N-k+1$ фиксировано, $s \geqslant 1$ (правая область);

3) $k=\lambda N+o(\sqrt{N}), \lambda \in(0,1)$ (средняя область).

Соответственно этой классификации получаются три типа предельных теорем для рассматриваемых статистик. Прежде чем формулировать эти теоремы, условимся о некоторых стандартных обозначениях.

Символом $\mathscr{L}(\xi)$ будем обозначать закон распределения случайной величины $\xi$; будем обозначать через $\operatorname{Bi}(n, p)$ биномиальньй закон распределения с параметрами $n$ и $p$, задаваемьй вероятностями

$$
b_{r}(n, p)=\left(\begin{array}{l}
n \\
r
\end{array}\right) p^{r}(1-p)^{n-r}, \quad r=0,1, \ldots, n, \quad p \in(0,1),
$$


и через $\Pi(t)$ пуассоновский закон распределения, задаваемьй вероятностями

$$
\pi_{r}(t)=e^{-t} \frac{t^{r}}{r !}, \quad r=0,1,2, \ldots, \quad t>0 .
$$

Пусть, далее,

$$
\beta(x ; k, N-k+1)=N\left(\begin{array}{c}
N-1 \\
k-1
\end{array}\right) x^{k-1}(1-x)^{N-k}, \quad x \in[0,1],
$$

есть бета-плотность, а

$$
B(z ; k, N-k+1)=\int_{0}^{z} \beta(x ; k, N-k+1) d x, \quad z \in[0,1],
$$

- соответствующая функция распределения; через $X_{r}$ ниже обозначается случайная величина, имеющая гамма-распределение с плотностью $\pi_{r-1}(x), x \geqslant 0$, и

$$
\Gamma_{r}(t)=\int_{0}^{t} \pi_{r-1}(x) d x, \quad t \geqslant 0
$$

- ее функция распределения.

Наконец, $\mathbb{I}(A)$ обозначает индикатор события $A$ (случайного или неслучайного), т.е. $\mathbb{I}(A)=1$, если $A$ имеет место, и $\mathbb{I}(A)=0$ в противном случае.

Асимптотическое поведение статистик $\bar{\mu}^{(\cdot)}$ и $\nu_{m}^{(\cdot)}(N, k)$ в левой области изменения параметра $k$ описывается следующей теоремой.

Теорема 1. Пусть $N \rightarrow \infty$, a $k \geqslant 1$ фиксировано. Тогда при $m=1$ для обеих моделей имеют место сходимости

$$
\mu_{1}^{(\cdot)} \stackrel{\mathrm{P}}{\longrightarrow} k, \quad \mu_{r}^{(\cdot)} \stackrel{\mathrm{P}}{\longrightarrow} 0, \quad r>1 .
$$

Если же $m>1($ и $m \leqslant$ а для бесповторного выбора), то

$$
\mu_{m}^{(\cdot)} \stackrel{\mathrm{P}}{\longrightarrow} k, \quad \mu_{r}^{(\cdot)} \stackrel{\mathrm{P}}{\longrightarrow} 0, \quad r>m
$$

а совместное распределение величин $\mu_{1}^{(\cdot)}, \ldots, \mu_{m-1}^{(\cdot)}$ удовлетворяет следующим предельным соотношениям:

$$
\mathscr{L}\left(\frac{\mu_{r}^{(\cdot)}}{c_{r, N}^{(\cdot)}}, r=1, \ldots, m-1\right) \rightarrow \mathscr{L}\left(X_{k}^{r / m}, r=1, \ldots, m-1\right),
$$

$2 \partial e$

$$
c_{r, N}^{(0)}=\frac{(m !)^{r / m}}{r !} N^{1-r / m}, \quad c_{r, N}^{(-)}=\left(\begin{array}{c}
a \\
r
\end{array}\right)\left(\begin{array}{c}
a \\
m
\end{array}\right)^{-r / m} N^{1-r / m} .
$$

Время ожидания $\nu_{m}^{(\cdot)}(N, k)$ асимптотически имеет такое же распределение, как $u \mu_{1}^{(\cdot)}$, m.e. $\nu_{1}^{(\cdot)}(N, k) \stackrel{\mathrm{P}}{\longrightarrow} k$, a npu $m>1$

$$
\mathscr{L}\left(\frac{\nu_{m}^{(\cdot)}(N, k)}{c_{1, N}^{(\cdot)}}\right) \rightarrow \mathscr{L}\left(X_{k}^{1 / m}\right)
$$


СлЕДСТВИЕ. Величина $\kappa_{N}^{(\cdot)}=\max \left\{r: \mu_{r}^{(\cdot)}>0\right\}$ в пределе вырождается $\boldsymbol{в}$ mочке $m: \kappa_{N}^{(\cdot)} \stackrel{\mathrm{P}}{\longrightarrow} m$.

Для правой области значений параметра $k$ имеет место следующее утверждение.

Tеорема 2. Пусть $N \rightarrow \infty$, a $s=N-k+1 \geqslant 1$ фиксировано. Тогда

$$
\mu_{r}^{(\cdot)} \stackrel{\mathrm{P}}{\longrightarrow} 0, \quad r<m-1, \quad \mu_{m-1}^{(\cdot)} \stackrel{\mathrm{P}}{\longrightarrow} s-1,
$$

а совместное распределение любого конечного числа величин $\mu_{r}^{(\cdot)}, r \geqslant m$, удовлетворяет следующим предельным соотношениям:

$$
\begin{gathered}
\mathscr{L}\left(\frac{\mu_{r}^{(0)}}{d_{r, N}^{(0)}}, r=m, \ldots, M\right) \rightarrow \mathscr{L}\left(X_{s}, \ldots, X_{s}\right), \\
\mathscr{L}\left(\frac{\mu_{r}^{(-)}}{d_{r, N}^{(-)}}, r=m, \ldots, a\right) \rightarrow \mathscr{L}\left(X_{s}^{(a-r) /(a-m+1)}, r=m, \ldots, a\right),
\end{gathered}
$$

$2 \partial e$

$$
\begin{aligned}
& d_{r, N}^{(0)}=\frac{(m-1) !}{r !}(\ln N)^{r-m+1} \\
& d_{r, N}^{(-)}=\left(\begin{array}{l}
a \\
r
\end{array}\right)\left(\begin{array}{c}
a \\
m-1
\end{array}\right)^{(r-a) /(a-m+1)} N^{(r-m+1) /(a-m+1)}
\end{aligned}
$$

Для времени ожсиания в модели повторного выбора справедливо соотношение

$$
\mathscr{L}\left(\frac{1}{N} \nu_{m}^{(0)}(N, k)-\ln N-(m-1) \ln \ln N+\ln (m-1) !\right) \rightarrow \mathscr{L}\left(-\ln X_{s}\right),
$$

в модели же бесповторного выбора при $m=$ а разность

$$
N a-\nu_{a}^{(-)}(N, k) \stackrel{\mathrm{P}}{\longrightarrow} s-1
$$

a npu $m<a$

$$
\mathscr{L}\left(\frac{1}{a}\left(\begin{array}{c}
a \\
m-1
\end{array}\right)^{1 /(a-m+1)} N^{(m-a) /(a-m+1)}\left(N a-\nu_{m}^{(-)}(N, k)\right)\right) \rightarrow \mathscr{L}\left(X_{s}^{1 /(a-m+1)}\right) .
$$


ЗАмЕчАниЕ. Для величины $\kappa_{N}^{(\cdot)}$ имеем $\kappa_{N}^{(-)} \stackrel{\text { P }}{\rightarrow} a$, в случае же повторного выбора известен следующий результат [3]:

$$
\mathrm{P}\left\{\kappa_{N}^{(0)}-A_{N} \leqslant j\right\}=\int_{0}^{\infty} \exp \left\{-\frac{e^{\varepsilon_{N}-j}}{c(m) x^{e-1}}\right\} \pi_{s-1}(x) d x+o(1), \quad j=0, \pm 1, \pm 2, \ldots
$$

где $c(m)=((m-1) !)^{e-1}(e-1) \sqrt{2 \pi e}$, а $A_{N}$ и $\varepsilon_{N}$ - целая и дробная части числа

$$
e \ln N+\left((m-1)(e-1)-\frac{1}{2}\right) \ln \ln N
$$

соответственно.

Для зоны средних значений $k$ характерна асимптотическая нормальность величин $\mu_{r}^{(\cdot)}$ и их линейных комбинаций, в частности, времени ожидания $\nu_{m}^{(\cdot)}(N, k)=\sum_{r} r \mu_{r}^{(\cdot)}$. Для формулировки соответствующих утверждений нам понадобятся дополнительные обозначения.

Ниже через $t_{m}(\lambda)$ обозначается $\lambda$-квантиль функции гамма-распределения $\Gamma_{m}(t)$, т.е. корень уравнения $\Gamma_{m}(t)=\lambda$; аналогично, $z_{m, a}(\lambda)$ есть $\lambda$-квантиль функции бета-распределения $B(z ; m, a-m+1): B\left(z_{m, a}(\lambda) ; m, a-m+1\right) \equiv \lambda, \lambda \in(0,1)$. Введем также случайные величины $\xi_{\lambda}^{(\cdot)}$ с распределениями

$$
\mathscr{L}\left(\xi_{\lambda}^{(0)}\right)=\Pi\left(t_{m}(\lambda)\right), \quad \mathscr{L}\left(\xi_{\lambda}^{(-)}\right)=\operatorname{Bi}\left(a, z_{m, a}(\lambda)\right)
$$

и положим

$$
p_{r}^{(\cdot)}(\lambda)=\mathrm{P}\left\{\xi_{\lambda}^{(\cdot)}=r\right\}= \begin{cases}\pi_{r}\left(t_{m}(\lambda)\right) & \text { для }(\cdot)=(0), \\ b_{r}\left(a, z_{m, a}(\lambda)\right) & \text { для }(\cdot)=(-) .\end{cases}
$$

Тогда справедлива

Теорема 3. Если $N, k \rightarrow \infty$ maк, что $k=\lambda N+o(\sqrt{N}), \lambda=1-\bar{\lambda} \in(0,1)$, mo совместное распределение любого конечного набора величин $\mu_{r}^{(\cdot)}$ асимптотически нормально со средними $\mathrm{E} \mu_{r}^{(\cdot)} \sim N p_{r}^{(\cdot)}(\lambda)$ и вторыми моментами $\operatorname{cov}\left(\mu_{r}^{(\cdot)}, \mu_{s}^{(\cdot)}\right) \sim$ $N \sigma_{r s}^{(\cdot)}(\lambda)$, əде

$$
\begin{aligned}
\sigma_{r s}^{(0)}(\lambda)= & p_{r}^{(0)}(\lambda)\left(\mathbb{I}(r=s)-p_{s}^{(0)}(\lambda)\right) \\
& +p_{r}^{(0)}(\lambda) p_{s}^{(0)}(\lambda)\left\{\lambda \overline { \lambda } ( \frac { t _ { m } ^ { \prime } ( \lambda ) } { t _ { m } ( \lambda ) } ) ^ { 2 } \left(r-t_{m}(\lambda)\left(s-t_{m}(\lambda)\right)\right.\right. \\
& \left.-\frac{t_{m}^{\prime}(\lambda)}{t_{m}(\lambda)}\left[\left(r-t_{m}(\lambda)\right)(\mathbb{I}(s \geqslant m)-\lambda)+\left(s-t_{m}(\lambda)\right)(\mathbb{I}(r \geqslant m)-\lambda)\right]\right\}, \\
\sigma_{r s}^{(-)}(\lambda)= & p_{r}^{(-)}(\lambda)\left(\mathbb{I}(r=s)-p_{s}^{(-)}(\lambda)\right) \\
& +p_{r}^{(-)}(\lambda) p_{s}^{(-)}(\lambda)\left\{\lambda \bar{\lambda}\left(\frac{z_{m, a}^{\prime}(\lambda)}{z_{m, a}(\lambda)\left(1-z_{m, a}(\lambda)\right)}\right)^{2}\left(r-a z_{m, a}(\lambda)\right)\left(s-a z_{m, a}(\lambda)\right)\right. \\
& -\frac{z_{m, a}^{\prime}(\lambda)}{z_{m, a}(\lambda)\left(1-z_{m, a}(\lambda)\right)}\left[\left(r-a z_{m, a}(\lambda)\right)(\mathbb{I}(s \geqslant m)-\lambda)\right. \\
& \left.\left.+\left(s-a z_{m, a}(\lambda)\right)(\mathbb{I}(r \geqslant m)-\lambda)\right]\right\},
\end{aligned}
$$


а время ожидания $\nu_{m}^{(\cdot)}(N, k)$ асимптотически нормально со средним

$$
\mathrm{E} \nu_{m}^{(\cdot)}(N, k) \sim N \mathrm{E} \xi_{\lambda}^{(\cdot)}= \begin{cases}N t_{m}(\lambda) & \partial \Omega_{\text {g }}(\cdot)=(0), \\ N a z_{m, a}(\lambda) & \partial \Omega_{\text {g }}(\cdot)=(-),\end{cases}
$$

u дисперсией $\mathrm{D} \nu_{m}^{(\cdot)}(N, k) \sim N \sigma^{(\cdot)^{2}}(\lambda)$, əде

$$
\begin{aligned}
\sigma^{(0)^{2}}(\lambda) & =\lambda \bar{\lambda}\left(t_{m}^{\prime}(\lambda)\right)^{2}-t_{m}(\lambda) \\
\sigma^{(-)^{2}}(\lambda) & =\lambda \bar{\lambda} a^{2}\left(z_{m, a}^{\prime}(\lambda)\right)^{2}-a z_{m, a}(\lambda)\left(1-z_{m, a}(\lambda)\right) .
\end{aligned}
$$

Приведем также для иллюстрации краткие таблицы значений квантильных функций $t_{m}(\lambda)$ и $z_{m, a}(\lambda)$, которые можно извлечь из сборника статистических таблиц [7].

ТАБлИцА 1. Значения $z_{m, a}(0.1)$ и $t_{m}(0.1)$

\begin{tabular}{|c|c|c|c|c|c|c|}
\hline 1 & 2 & 3 & 4 & 5 & 6 & $m / a$ \\
\hline \hline 0.100 & & & & & & 1 \\
\hline 0.051 & 0.316 & & & & & 2 \\
\hline 0.035 & 0.196 & 0.464 & & & & 3 \\
\hline 0.026 & 0.143 & 0.320 & 0.562 & & & 4 \\
\hline 0.021 & 0.112 & 0.247 & 0.416 & 0.631 & & 5 \\
\hline 0.017 & 0.093 & 0.201 & 0.333 & 0.490 & 0.681 & 6 \\
\hline 0.106 & 0.532 & 1.102 & 1.745 & 2.433 & 3.152 & $t_{m}(0.1)$ \\
\hline
\end{tabular}

ТАБлицА 2. Значения $z_{m, a}(0.5)$ и $t_{m}(0.5)$

\begin{tabular}{|c|c|c|c|c|c|c|}
\hline 1 & 2 & 3 & 4 & 5 & 6 & $m / a$ \\
\hline \hline 0.500 & & & & & & 1 \\
\hline 0.293 & 0.707 & & & & & 2 \\
\hline 0.206 & 0.500 & 0.794 & & & & 3 \\
\hline 0.159 & 0.386 & 0.614 & 0.841 & & & 4 \\
\hline 0.129 & 0.314 & 0.500 & 0.686 & 0.871 & & 5 \\
\hline 0.109 & 0.264 & 0.421 & 0.579 & 0.736 & 0.891 & 6 \\
\hline 0.693 & 1.678 & 2.674 & 3.672 & 4.671 & 5.670 & $t_{m}(0.5)$ \\
\hline
\end{tabular}

ТАБлИцА 3. Значения $z_{m, a}(0.9)$ и $t_{m}(0.9)$

\begin{tabular}{|c|c|c|c|c|c|c|}
\hline 1 & 2 & 3 & 4 & 5 & 6 & $m / a$ \\
\hline \hline 0.900 & & & & & & 1 \\
\hline 0.684 & 0.949 & & & & & 2 \\
\hline 0.536 & 0.804 & 0.965 & & & & 3 \\
\hline 0.438 & 0.680 & 0.857 & 0.974 & & & 4 \\
\hline 0.369 & 0.584 & 0.753 & 0.888 & 0.979 & & 5 \\
\hline 0.319 & 0.510 & 0.667 & 0.799 & 0.907 & 0.983 & 6 \\
\hline 2.302 & 3.889 & 5.323 & 6.681 & 7.994 & 9.275 & $t_{m}(0.9)$ \\
\hline
\end{tabular}


3. Об идентификации модели. В этом пункте мы обсудим вопрос о возможности идентификации модели выбора, основьваясь на изложенных вьше результатах. В статистической терминологии речь идет о различении гипотез $H^{(0)}$ и $H^{(-)}$, означающих соответственно, что выбор осуществлялся по схеме повторной и бесповторной выборки. Прежде всего отметим, что при гипотезе $H^{(0)}$ параметр $а$ никак не сказьвается на распределении статистик $\mu_{r}$ и $\nu_{m}(N, k)$; это является отражением того факта, что вероятность появления каждого из цветов в любом испытании равна $a / N a=1 / N$, т.е. не зависит от $a$. Тем самым, если требуется сделать те или иные статистические заключения о составе совокупности (о параметре $a$ ), то это в принципе невозможно при повторном выборе, и соотвествующий статистический эксперимент следует организовать с использованием механизма бесповторного выбора.

Обратимся теперь к конкретным ситуациям, характеризуемьм значениями параметров $k$ и $m$, считая, что $N$ велико.

1) Если $k \geqslant 1$ фиксировано, а $m=1$, то между двумя моделями выбора фактически никакой разницы наблюдаться не будет: в обоих случаях с вероятностью, близкой к 1 , потребуется произвести всего лишь $k$ испытаний, и при этом в них будут зафиксированы $k$ различных цветов (см. (1)), т.е. для различения гипотез $H^{(0)}$ и $H^{(-)}$информации в данном случае недостаточно. Если же при фиксированном $k$ параметр $m>1$, то, как следует из (3), статистики $\mu_{r}, r=1, \ldots, m-1$, будут вести себя асимптотически по-разному в зависимости от того, какая из гипотез $H^{(0)}$ или $H^{(-)}$истинна, т.е. эти статистики, а также статистика $\nu_{m}(N, k)($ см. (4)), могут быть использованыв качестве тестовых статистик для различения этих гипотез. При этом наиболее информативной является, очевидно, статистика $\mu_{1}$, либо асимптотически эквивалентная ей статистика $\nu_{m}(N, k)$, поэтому, основьваясь на соотношении (4), можно предложить следующий статистический критерий для различения гипотез $H^{(0)}$ и $H^{(-)}$: если наблюдается событие $\left\{\nu_{m}(N, k) \leqslant c_{1, N}^{(0)} t_{\alpha}\right\}$, то принимается гипотеза $H^{(0)}$, в противном случае принимается гипотеза $H^{(-)}$. Если здесь $t_{\alpha}$ задать так, чтобы выполнялось условие

$$
\Gamma_{k}\left(t_{\alpha}^{m}\right)=1-\alpha, \quad \alpha \in(0,1)
$$

то вероятность ошибки первого рода (отклонить $H^{(0)}$, когда она верна) будет удовлетворять в силу (4) соотношениям

$$
\mathrm{P}^{(0)}\left\{\nu_{m}(N, k)>c_{1, N}^{(0)} t_{\alpha}\right\} \rightarrow \mathrm{P}\left\{X_{k}^{1 / m}>t_{\alpha}\right\}=1-\Gamma_{k}\left(t_{\alpha}^{m}\right)=\alpha .
$$

Вероятность же ошибки второго рода (принять $H^{(0)}$, когда истинной является гипотеза $\left.H^{(-)}\right)$при этом такова:

$$
\mathrm{P}^{(-)}\left\{\nu_{m}(N, k) \leqslant c_{1, N}^{(0)} t_{\alpha}\right\}=\mathrm{P}^{(-)}\left\{\frac{\nu_{m}(N, k)}{c_{1, N}^{(-)}} \leqslant \frac{(a)_{m}^{1 / m}}{a} t_{\alpha}\right\} \rightarrow \Gamma_{k}\left(\frac{(a)_{m}}{a^{m}} t_{\alpha}^{m}\right)<1-\alpha
$$

т.е. критерий является несмещенным (здесь $\left.(a)_{m}=a(a-1) \cdots(a-m+1)\right)$.

Итак, при фиксированном $k$ и $m>1$ существует критерий, основанньй на времени ожидания $\nu_{m}(N, k)$, позволяющий различать гипотезы $H^{(0)}$ и $H^{(-)}$с ошибками, указанными в $(14),(15)$. Отметим также, что с увеличением уровня $m$ предельная ошибка 
в (15) убывает, в то время как с ростом параметра $a$ она возрастает (различение гипотез затрудняется).

2) Обсудим аналогично второй крайний случай, когда параметр $k$ принимает значения, близкие к $N$. Теперь мы основываемся на теореме 2 , из которой следует, что информативными статистиками являются здесь величины $\mu_{r}$ при $r \geqslant m$ и время ожидания $\nu_{m}(N, k)$. При этом наиболее рельефно различие меж ду моделями проявляется в характере асимптотического поведения последней статистики: если при гипотезе $H^{(0)}$ время ожидания растет как $N \ln N$, то в случае бесповторного выбора оно имеет порядок $N a$. Поэтому данная статистика вполне идентифицирует модель, а соответствуюшее правило принятия решения может быть сформулировано, например, следующим образом: если $\nu_{m}(N, k)>N(\ln N-\ln \ln N)$, то принимается гипотеза $H^{(0)}$, в противном случае принимается гипотеза $H^{(-)}$. Вероятности ошибок первого и второго рода такого правила удовлетворяют в силу $(8),(9)$ соотношениям

$$
\begin{aligned}
\mathrm{P}^{(0)} & \left\{\nu_{m}(N, k) \leqslant N(\ln N-\ln \ln N)\right\} \\
= & \mathrm{P}^{(0)}\left\{\frac{1}{N} \nu_{m}(N, k)-\ln N-(m-1) \ln \ln N+\ln (m-1) !\right. \\
\quad \leqslant-m \ln \ln N+\ln (m-1) !\} \rightarrow 0, & \mathrm{P}^{(-)}\left\{\nu_{m}(N, k)>N(\ln N-\ln \ln N)\right\} \\
= & \mathrm{P}^{(-)}\left\{N^{(m-a) /(a-m+1)}\left(N a-\nu_{m}(N, k)\right)\right. \\
& \left.<N^{(m-a) /(a-m+1)}(N a-N(\ln N-\ln \ln N))\right\} \rightarrow 0 .
\end{aligned}
$$

Таким образом, этот критерий позволяет асимптотически достоверно различать гипотезы $H^{(0)}$ и $H^{(-)}$.

Аналогичные тесты можно построить и на основании статистик $\mu_{r}, r \geqslant m$. Отметим также, что статистика $\kappa_{N}$ - максимальная частота повторения цветов в выборкев модели бесповторного выбора при рассматриваемых условиях асимптотически достоверно идентифицирует параметр $a$. В модели же повторного выбора она неограниченно растет как $e \ln N$ (см. (10)), поэтому данная статистика также асимптотически достоверно различает гипотезы $H^{(0)}$ и $H^{(-)}$.

3) Рассмотрим, наконец, зону средних значений параметра $k$. Теорема 3 в этом случае утверждает, что все наши статистики при обеих гипотезах асимптотически нормальны, при этом в случае справедливости гипотезы $H^{(\cdot)}$

$$
\frac{\mu_{r}}{N} \stackrel{\mathrm{P}}{\longrightarrow} p_{r}^{(\cdot)}(\lambda), \quad \frac{\nu_{m}(N, k)}{N} \stackrel{\mathrm{P}}{\longrightarrow} \mathrm{E} \xi_{\lambda}^{(\cdot)}
$$

(см. соотношения $(11),(12))$. Поскольку здесь пределы при гипотезах $H^{(0)}$ и $H^{(-)}$различаются, то каждая из этих статистик асимптотически достоверно идентифицирует модель. Например, если использовать в качестве тестовой статистики время ожидания $\nu_{m}(N, k)$ (параметры этой статистики рассчитываются наиболее просто), то при $t_{m}(\lambda)>a z_{m, a}(\lambda)($ см. $(12))$ критическую область для гипотезы $H^{(0)}$ можно задать в виде

$$
\left\{\nu_{m}(N, k)<N t_{m}(\lambda)-\sqrt{N} \ln N\right\}
$$


а при $t_{m}(\lambda)<a z_{m, a}(\lambda)-$ в виде

$$
\left\{\nu_{m}(N, k)>N t_{m}(\lambda)+\sqrt{N} \ln N\right\} .
$$

С использованием теоремы 3 легко убедиться в том, что вероятности ошибок первого и второго рода такого критерия будут стремиться при $N \rightarrow \infty$ к нулю, т.е. гипотезы $H^{(0)}$ и $H^{(-)}$различаются асимптотически достоверно.

ЗАмЕчАниЕ. Вьше уже отмечалось, что с увеличением параметра $a$ различение гипотез $H^{(0)}$ и $H^{(-)}$затрудняется. Если мы находимся в условиях теоеремы 3 , то нетрудно видеть, что при фиксированных $\lambda$ и $m$ квантиль $z_{m, a}(\lambda) \rightarrow 0$, когда $a \rightarrow \infty$, a $a z_{m, a}(\lambda) \rightarrow t_{m}(\lambda)$. Это следует из цепочки соотношений

$$
\begin{aligned}
\lambda & =\int_{0}^{z_{m, a}(\lambda)} \beta(x ; m, a-m+1) d x=\int_{0}^{a z_{m, a}(\lambda)} \beta\left(\frac{y}{a} ; m, a-m+1\right) \frac{d y}{a} \\
& \sim \int_{0}^{a z_{m, a}(\lambda)} \pi_{m-1}(y) d y=\Gamma_{m}\left(a z_{m, a}(\lambda)\right) .
\end{aligned}
$$

В этом случае асимптотические распределения статистики $\nu_{m}(N, k)$ при гипотезах $H^{(0)}$ и $H^{(-)}$сближаются; следовательно, различение гипотез затрудняется. Интересно было бы исследовать вопрос, с какой скоростью может возрастать $a=a(N)$, чтобы еще было возможньм различение гипотез $H^{(0)}$ и $H^{(-)}$с некоторьми ошибками. По-видимому, это будет так, если $a \asymp \sqrt{N}$.

4. О методе доказательства предельных теорем. Пусть $\eta_{j}, j=1, \ldots, N,-$ частоты соответствующих цветов в выборке в момент остановки испытаний и $g(x)-$ произвольная функция. Случайная величина вида

$$
L_{N k}=\sum_{j=1}^{N} g\left(\eta_{j}\right)
$$

назьвается разделимой статистикой (РС). Разделимая статистика - весьма общий объект, охватьваюший широкий класс характеристик в рассматриваемых моделях выбора. Частными случаями разделимых статистик являются обсуждаемые нами величины $\mu_{r}$ (при $g(x)=\mathbb{I}(x=r)$ ) и $\nu_{m}(N, k)$ (при $\left.g(x)=x\right)$. Общий метод исследования асимптотических (при $N \rightarrow \infty$ и $k=k(N)$ ) распределений разделимьх статистик для общей модели выбора (модели Маркова-Пойа), включающей как частные случаи и модели повторного и бесповторного выбора, развит в работах [1], [4]-[6]. Суть этого метода состоит в следующем: с помощю приема вложения урновой схемы в подходящий марковский процесс с непрерьвным временем выводится общее интегральное представление для характеристической функции разделимой статистики (17), которое и является основой для получения предельных распределений разделимых статистик при $N \rightarrow \infty$.

Чтобы выписать это представление для рассматриваемой нами ситуации, введем случайные величины $\xi^{(\cdot)}(t)$ с распределениями

$$
\mathscr{L}\left(\xi^{(\cdot)}(t)\right)=\left\{\begin{array}{ll}
\Pi(a t) & \text { для }(\cdot)=(0), \\
\operatorname{Bi}\left(a, 1-e^{-t}\right) & \text { для }(\cdot)=(-),
\end{array} \quad t>0,\right.
$$


и соответствуюшие усеченные случайные величины $\xi_{j}^{(\cdot)}(t), j=1,2$, с распределениями

$$
\mathscr{L}\left(\xi_{1}^{(\cdot)}(t)\right)=\mathscr{L}\left(\xi^{(\cdot)}(t) \mid \xi^{(\cdot)}(t) \geqslant m\right), \quad \mathscr{L}\left(\xi_{2}^{(\cdot)}(t)\right)=\mathscr{L}\left(\xi^{(\cdot)}(t) \mid \xi^{(\cdot)}(t)<m\right)
$$

а также характеристические функции

$$
f_{j}^{(\cdot)}(\tau ; t)=\mathrm{E} \exp \left\{i \tau g\left(\xi_{j}^{(\cdot)}(t)\right)\right\}, \quad j=1,2
$$

Тогда

$$
\mathrm{E} e^{i \tau L_{N k}^{(\cdot)}}=e^{i \tau g(m)} \int_{0}^{1} f_{N k}^{(\cdot)}\left(\tau ; t_{m}^{(\cdot)}(x)\right) \beta(x ; k, N-k+1) d x
$$

где

$$
f_{N k}^{(\cdot)}(\tau ; t)=\left[f_{1}^{(\cdot)}(\tau ; t)\right]^{k-1}\left[f_{2}^{(\cdot)}(\tau ; t)\right]^{N-k}
$$

и $t_{m}^{(\cdot)}(x), x \in[0,1],-$ обратная функция к

$$
H_{m}^{(\cdot)}(t)=\mathrm{P}\left\{\xi^{(\cdot)}(t) \geqslant m\right\}
$$

С помощью представления (18) в цитируемых работах получены предельные при $N \rightarrow \infty$ распределения произвольной разделимой статистики для каждой из трех зон изменения параметра $k$.

Здесь мы рассматриваем совместные распределения величин $\mu_{r}$, поэтому прямо использовать известные предельные теоремы для разделимых статистик, чтобы получить нужные нам многомерные распределения, нельзя. Тем не менее, описанная схема работает и в многомерном случае. Именно, если ввести функцию

$$
g(\bar{\tau} ; x)=\sum_{r} \tau_{r} \mathbb{I}(x=r)
$$

то соответствующая разделимая статистика (17) примет вид

$$
L_{N k}=\sum_{j=1}^{N} g\left(\bar{\tau} ; \eta_{j}\right)=\sum_{r} \tau_{r} \sum_{j=1}^{N} \mathbb{I}\left(\eta_{j}=r\right)=\sum_{r} \tau_{r} \mu_{r}
$$

а ее характеристическая функция в единице $\mathrm{E} \exp \left\{i L_{N k}\right\}=\operatorname{E} \exp \left\{i \sum_{r} \tau_{r} \mu_{r}\right\}$ как функция параметров $\bar{\tau}=\left\{\tau_{r}\right\}$ и будет совместной характеристической функцией величин $\mu_{r}$. Следовательно, если в (18) функция $g$ имеет вид (19), то мы можем использовать все результаты для общих разделимых статистик, выводя из них соответствующие утверждения для совместных распределений величин $\mu_{r}$. Реализацией этой идеологии и являются приведенные в п. 2 теоремы. Технические детали ввиду их громоздкости мы опускаем, а все ключевые элементы соответствующей аналитики можно извлечь из работы [1]. Отметим также, что утверждения теорем 2 и 3 о времени ожидания в модели повторного выбора известны еще из работы [8], а утверждение (8) для случая $s=1$ впервые было получено в [9]. 


\section{СПИСОК ЦИТИРОВАННОЙ ЛИТЕРАТУРЫ}

[1] Ivanov A. V., Ivchenko G.I. On waiting time in the Markov-Pólya scheme // J. Math. Sci. 1998. V. 91. №3. P. 2904-2916.

[2] Ивченко Г.И. Время ожидания и связанные с ним характеристики в полиномиальной схеме // Дискретная матем. 1993. Т. 5. № 3. С. 3-34.

[3] Ивченко Г.И., Медведев Ю.И. Смеси вероятностных распределений и случайные размещения // Тр. по дискретной матем. 1998. Т. 2. С. 169-182.

[4] Ивченко Г.И., Иванов А. В. Разделимые статистики в обратных урновых задачах // Дискретная матем. 1995. Т. 7. № 2. С. 103-117.

[5] Иванов А. В., Ивченко Г. И. Разделимые статистики и моменты остановки в схеме бесповторного выбора // Дискретная матем. 1997. Т. 9. №1. С. 43-58.

[6] Holst L., Hüsler J. Sequential urn schemes and birth processes // Adv. Appl. Probab. 1985. V. 17. P. 257-279.

[7] Большев Л. Н., Смирнов Н. В. Таблицы математической статистики. М.: Наука, 1983.

[8] Bekessy A. On classical occupancy problems. II // Magy. Tud. Akad. Mat. Kutató Int. Közl. 1964. V. 9A. №1-2. P. 133-141.

[9] Erdös P., Renyi A. On a classical problem of probability theory // Magy. Tud. Akad. Mat. Kutató Int. Közl. 1961. V. 6A. №1-2. P. 215-220.

Московский государственный институт электроники и математики

Поступило

25.10 .2001 